\title{
Emergency support of transmission voltages by active distribution networks: a non-intrusive scheme
}

\author{
Luis David Pabón Ospina, Member, IEEE, and Thierry Van Cutsem, Fellow, IEEE
}

\begin{abstract}
Non-negligible amounts of Distributed Generating Units (DGUs) are already connected to power systems, predominantly to medium- and low-voltage networks. The interaction between distribution and transmission systems is gaining the attention of system operators. Given the high controlability of DGUs, the active distribution networks can potentially support the transmission system during emergency situations. Previously proposed voltage emergency controls involving distribution network assets focused on preserving the transmission system integrity, even if it implies affecting the distribution system operation (intrusive schemes). More attention needs to be devoted to supporting transmission voltages, while preserving the distribution network operation (non-intrusive schemes). This paper introduces a new adaptive and non-intrusive voltage emergency control scheme based on the synchronization of DGUs and the load tap changer of the corresponding distribution transformer. The conclusions are derived from time-domain simulations using an extended version of the IEEE Nordic test system for voltage stability assessment.
\end{abstract}

Index Terms-Active distribution networks, distributed generation, emergency control, load voltage reduction, voltage stability.

\section{INTRODUCTION}

$\mathbf{P}$ REVIOUSLY proposed voltage emergency control schemes focused on preserving the transmission system integrity, even if it implies affecting or over-using the Distribution Network (DN) assets. For instance, a commonly used scheme is the load-side voltage reduction, also known as conservation voltage reduction [1]. This emergency control exploits the voltage sensitivity of loads by intentionally reducing the distribution voltage to a predefined value (e.g. $0.95 \mathrm{pu}$ ) in emergency conditions. In this case, the DN is affected by a predefined voltage reduction in order to alleviate the transmission system stress. In references [2] and [3], the effectiveness of reducing the voltage setpoint of the Load Tap Changers (LTC) of distribution transformers during system emergencies is illustrated. It is shown that, assuming load sensitivity to voltage, system instability is avoided when the distribution voltages in a large enough area are reduced by $5 \%$. The same technique is used in [1] in order to reduce peak demands and energy consumption.

In Germany, for instance, it is stated in [4] that during voltage emergency situations, "it is crucial that the transformers

Luis Pabón is with the Department of System Stability and Grid Integration, Fraunhofer IEE, Kassel, 34119 Germany e-mail: luis.david.pabon.ospina@iee.fraunhofer.de.

Thierry Van Cutsem is with the Fund for Scientific Research (FNRS) at the Dept. of Electrical Engineering and Computer Science, University of Liège, Belgium; e-mail: t.vancutsem@uliege.be. of all voltage levels are blocked". "Furthermore, the voltage in the distribution networks should be lowered to the permissible limits."

In Ref. [5], the main challenges associated with high shares of Distributed Generating Units (DGUs) are discussed. Voltage issues under normal operation are highlighted. Furthermore, [5] discusses different control techniques in active distribution grids such as local control, decentralized control, distributed control, and centralized control. The performance of the different techniques is compared in terms of their optimality, flexibility, need for communication and ability to maintain adequate load-side voltages. Conversely, this work proposes a voltage emergency control scheme that focuses on emergency situations only, complementing the above-mentioned control techniques. Avoiding a potential system collapse while preserving the adequate DN operation becomes a higher priority than optimizing resources, in emergency situations.

Due to the increased number of DGUs, there have been recent efforts towards exploiting their relatively high controlability in order to improve long-term voltage stability. This is one aspect of the emergence of the so-called Active Distribution Network (ADN).

References [6] and [7], for instance, study the possibility of DGUs injecting reactive power after an alarm of potential voltage instability is received. It is concluded that, this approach actually brings along counterproductive effects that may even precipitate voltage instability. On the contrary, the same studies emphasize that reducing, or even consuming reactive power with the DGUs, improves long-term voltage stability in so far as the load voltage is decreased, thus reducing the load consumption and alleviating the system stress.

The more severe the system emergency, the more intrusive the remedial actions. One good example is load shedding, which is used as the last line of defense against voltage instability. In reference [8], a load shedding scheme to counteract voltage instability is proposed. It uses the voltage sensitivity to reactive power variations as an index to decide the shedding location and amount that stabilizes the system. In reference [9], the load shedding scheme relies on a set of distributed controllers, each monitoring a transmission voltage, controlling a group of loads and adjusting its action to the voltage evolution. In reference [10], the undervoltage load shedding is based on wide-area monitoring. Voltage sensitivity analysis is considered to determine the shedding location and amount. Although those studies focus on minimizing the curtailed load power, regardless the efforts, this is the most intrusive scheme.

As more DGUs are connected to DNs, the emergency control schemes need to become less intrusive and more 


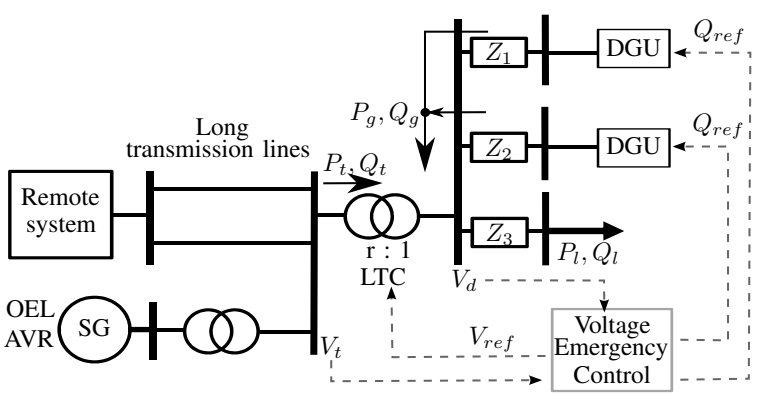

Fig. 1. Conceptual diagram of a simple transmission system and an ADN.

attention must be paid to supporting system stability while preserving the DN integrity. Intrusive actions such as load-side voltage reduction, may even become detrimental as the number of DGUs increases due to higher risk of DGU disconnection. In this paper, a voltage emergency control scheme involving ADNs is presented. It enhances long-term voltage stability while avoiding to affect or over-use the DN assets.

The remaining of this paper is structured as follows: Section II explains the trade-offs of state-of-the-art voltage emergency controllers with reference to the power space portrait. Section III details the proposed emergency control scheme. Sections IV and V present the test systems and the simulation results, respectively. The most relevant findings of this work are summarized in Section VI.

\section{TRADE-OFFS IN EMERGENCY CONTROL OF TRANSMISSION VOLTAGE INVOLVING ADNS}

Let us consider a DN feeding loads and hosting DGUs, together with a simple transmission system, as shown in Fig. 1.

Representing the transmission-generation system as a Thévenin equivalent consisting of a voltage source $E$ behind a reactance $X$, the feasible region, i.e. the set of $\left(P_{t}, Q_{t}\right)$ combinations for which the system can operate in steady state is given by [11]:

$$
-\left(\frac{P_{t} X}{E^{2}}\right)^{2}-\frac{Q_{t} X}{E^{2}}+\frac{1}{4} \geq 0
$$

This is illustrated with the solid or dashed line in Fig. 2, showing the boundary of the feasible region. A long-term equilibrium outside the feasible region does not exist [11] and any attempt of bringing the $\left(P_{t}, Q_{t}\right)$ powers outside this region results in voltage instability.

Let us now assume that the tripping of one of the two transmission lines (see Fig. 1) results in voltage instability. Before the line outage, the power consumption is at the longterm equilibrium shown with a black dot in Fig. 2, inside the feasible region bounded by the dashed curve. After the line outage, the maximum power that the combined transmissiongeneration system can supply is reduced. Therefore, the feasible region shrinks (see solid line). Due to the load sensitivity to voltage, the power consumption $\left(P_{t}, Q_{t}\right)$ moves to a postcontingency operating point shown with a circle in Fig. 2. It should be noted that this is not a long-term equilibrium. Subsequently, this point will move under the effect of the

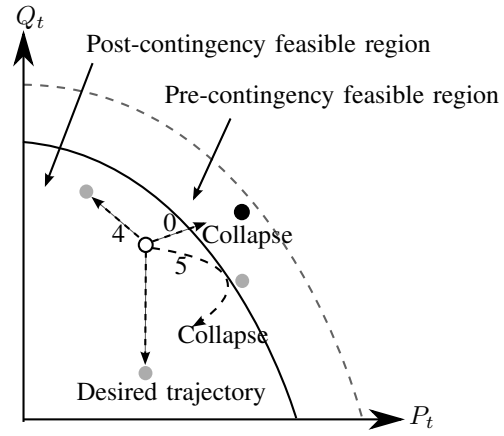

Fig. 2. The power space.

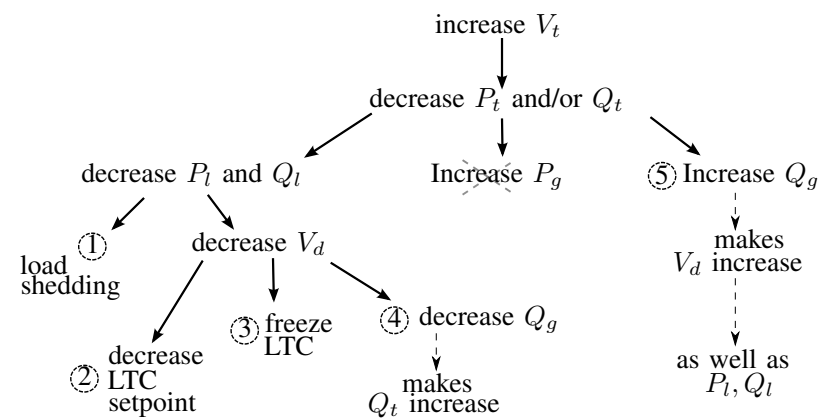

Fig. 3. Trade-offs of voltage emergency controls.

load dynamics and the emergency control strategy towards a targeted long-term equilibrium (grey dots). If the latter dynamics are such that the power consumption tends to be restored outside the new feasible region, the system will experience long-term voltage instability. Let us take trajectory 0 in Fig. 2 as an example. This trajectory corresponds to the natural instability mechanism without any emergency control. Typically, the LTC attempts to increase $V_{d}$, i.e. to restore the power consumption to its pre-disturbance long-term equilibrium value, which is infeasible.

Let us now consider an emergency control aimed at counteracting voltage instability. Five such control schemes are sketched in Fig. 3. Note that they all have the same goal: increase the transmission voltage $V_{t}$. This can be achieved by decreasing $P_{t}$ and/or $Q_{t}$, which, in turn, can be done by:

- reducing the load consumption $P_{l}$ and $Q_{l}$, or

- increasing the active power generation $P_{g}$, or

- increasing the reactive power generation $Q_{g}$.

Control 1, the load shedding, is one of the most effective countermeasures but intrusive, as discussed in the Introduction. Control 2, the LTC voltage setpoint reduction may not be sufficient, unless a large reduction of the $V_{d}$ voltage is applied. Therefore, it negatively affects the ADN and becomes also an intrusive scheme. A related technique, Control 3, consists in freezing the LTC in order to stop the instability mechanism and avoid a further decrease of transmission voltages. It implies that the ADN loses its voltage control capability and does not react to further transmission voltage drops. Controls 2 and 3 are common practices among system operators.

Control 4, used for instance in Refs. [6], [7], consists in reducing the DGU reactive power injections in order to reduce 
the load-side voltage. Even though it succeeds in decreasing $P_{t}$, the DGUs reduce or may even consume reactive power, leading to a counterproductive increase of $Q_{t}$ as shown by trajectory 4 in Fig. 2, along which $P_{t}$ decreases but $Q_{t}$ increases. The figure suggests that in some cases the targeted equilibrium point could leave the feasible region.

Another way of decreasing the power delivered by the transmission system, is to increase the DGU active power generation $P_{g}$. This is seldom an option, for two reasons: 1) most DGUs exploiting renewable energy sources operate at maximum power output, and 2) it relies on the availability of intermittent energy sources such as solar irradiation. Therefore it is not considered in this work.

A third option aimed at decreasing the reactive power delivered by the transmission system consists in increasing the reactive power $Q_{g}$ injected by the DGUs. This action effectively reduces $Q_{t}$, but it leads to an increase of the load-side voltage $V_{d}$, causing an undesired increase of load consumption that results in a counterproductive increase of $P_{t}$. This is depicted by trajectory 5 in Fig. 2, which corresponds to a corrective control failure, the targeted equilibrium being still outside the feasible region. As noticed in Refs. [6], [7], this counterproductive effect may even precipitate voltage instability, while making it less predictable.

By way of summary, a desirable trajectory in the power space is to move away from the border of the feasible region by reducing $P_{t}$ and $Q_{t}$. But reducing $P_{t}$ implies:

- curtailing load (not desired), or

- significantly reducing load-side voltage (not desired), or

- increasing the active power of DGUs (not an option).

Therefore, a desired trajectory corresponds to decreasing $Q_{t}$ without significantly affecting the load-side voltage or causing undesired load restoration. This means moving in a downwards trajectory as shown in Fig. 2.

\section{PROPOSED EMERGENCY CONTROL SCHEME}

\section{A. Design criteria}

The control actions of the proposed scheme are based on two input signals: the transmission voltage $V_{t}$ and the distribution voltage $V_{d}$ as depicted in Fig. 1. The outputs are the reactive power commands for the DGUs and the voltage setpoint for the transformer LTC. The control is designed to meet the following characteristics:

1) Non-intrusive. The scheme should support transmission voltages without exaggeratedly affecting the DN voltages or over-using its assets.

2) Adaptive. The control should adapt to the severity of the disturbance at transmission level, i.e. less critical situations should lead to milder control actions.

3) Local. The scheme should not require a dedicated exchange of information with transmission nor between ADNs (which would be impractical in several respects).

4) The number of inputs should be small to simplify the implementation.

5) The control should be effective regardless of the transmission system characteristics at the ADN connection point,

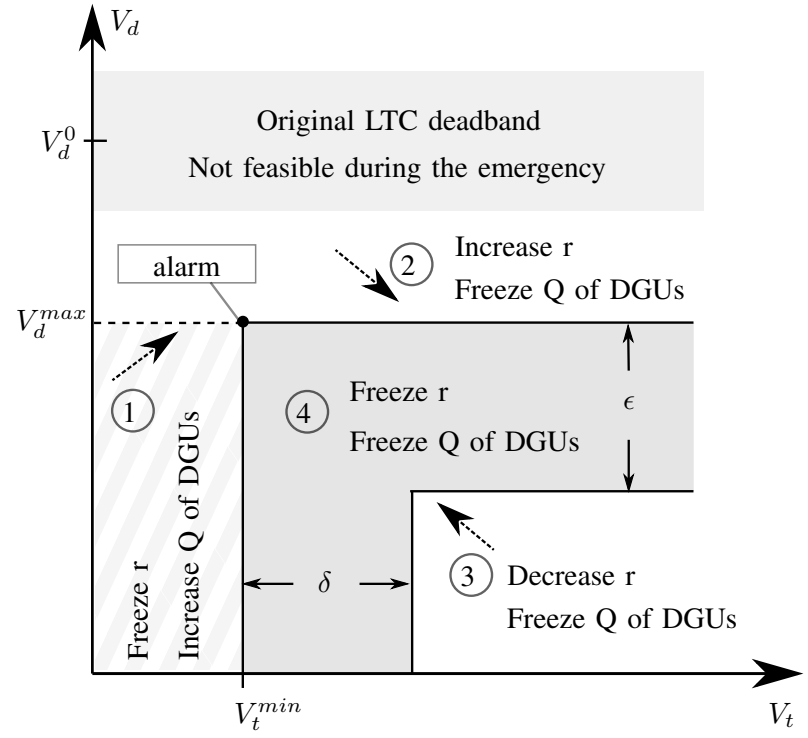

Fig. 4. Proposed control scheme in the $\left(V_{t}, V_{d}\right)$ space.

e.g. short circuit power or voltage sensitivity to reactive power variations.

6) The control should be effective regardless of the ADN load characteristics, i.e. type of loads and their dependency to voltage.

\section{B. Control scheme}

The control decisions are based on a diagram distinguishing four operating modes, based on the measured transmission and distribution voltages. From here on, this diagram is referred to as the $\left(V_{t}, V_{d}\right)$ space. It is shown in Fig. 4.

Two control actions are associated to each of the four areas in the $\left(V_{t}, V_{d}\right)$ space. The dashed arrows besides the area numbers denote the expected overall trajectory after applying the control actions of the corresponding area.

When a potential voltage instability is detected, the control stores the current values of $V_{t}$ and $V_{d}$. These two voltages at the moment of alarm will be referred to as $V_{t}^{\min }$ and $V_{d}^{\max }$ respectively. The objective of the emergency voltage control is twofold:

(i) the transmission voltage should not fall below its value at the moment of alarm $\left(V_{t}>V_{t}^{\text {min }}\right) . V_{t}^{\text {min }}$ is considered as a critical voltage level and efforts are directed towards staying above this critical value;

(ii) no attempt should be made to rise the distribution voltage above its value at the moment of alarm $\left(V_{d}<V_{d}^{\max }\right)$ to prevent load power from recovering above its value at that moment.

With this basic premise, let us now analyze each of the four areas in Fig. 4.

Area 1: when operating inside this area, condition (i) above is not met. Therefore the following actions are taken:

- freeze the LTC. This stops the load power restoration responsible for the decrease of $V_{t}$;

- increase DGU reactive powers. This is aimed at injecting reactive power into the transmission grid to raise its 
voltage or at least reduce the reactive power that the transmission system delivers to the DN. It can be also seen as power factor improvement at the point of coupling with transmission.

As a result of these two control actions, the operating point in the $\left(V_{t}, V_{d}\right)$ space could move into either Area 2 or Area 4.

Area 2: when operating inside this area, condition (ii) above is not met. Therefore the following actions are taken:

- freeze the DGU reactive power injection. This puts an end to the counterproductive effect of load restoration under the effect of increasing voltage;

- increase the transformer ratio ( $r$ in Fig. 1). This reduces $V_{d}$ to the desired range (below $V_{d}^{\max }$ ) and favours the increase of $V_{t}$.

The resulting effect is a movement of the operating point into Area 4.

Area 3: an operating point inside this area means that the transmission voltage has recovered above $V_{t}^{\text {min }}+\delta$. While beneficial for the transmission system, this has been obtained at the cost of lowering the DN voltages below $V_{d}^{\max }-\epsilon$. At this point, an effort should be made to preserve DN operation. Consequently, the following two actions are applied:

- freeze the DGU reactive power injection. Since $V_{t}$ has recovered a good value, the DGUs should not be overused;

- decrease the transformer ratio. This increases $V_{d}$ to the desired range. Note that, this could be done with the DGUs as well, but the LTC is preferred to avoid abusing the support of DGUs.

The resulting effect is a movement of the operating point into Area 4.

Area 4: No further action is taken when the operating point $\left(V_{t}, V_{d}\right)$ stays inside this "dead zone". The best location inside Area 4 is the sub-area defined by $V_{t}^{\text {min }}<V_{t}<V_{t}^{\text {min }}+\delta$ and $V_{d}^{\max }-\epsilon<V_{d}<V_{d}^{\max }$. Indeed, then, the distribution voltage is just below $V_{d}^{\max }$ (hence, impact on load is minimal) while the transmission voltage $V_{t}$ is just above the alarm threshold $V_{t}^{\text {min }}$ (indicating that minimal actions have been taken to restore the transmission voltage).

One could expect that freezing the transformer ratio and the DGUs reactive power injections ensures operation inside Area 4. Nevertheless, it should be noted that $V_{t}$ and $V_{d}$ are also affected by events taking place in other parts of the system. For instance, the OverExcitation Limiter (OEL) of a nearby synchronous generator may operate. The generator voltage being no longer controlled, $V_{t}$ and $V_{d}$ will decrease, moving the operating point into Area 1. If this is the case, the control actions corresponding to Area 1 are applied again until the operating point re-enters Area 4. Thus, the control scheme adapts to the evolving severity of the event.

Note that the voltage sensitivities to reactive power are different from one DGU to another. This is because of the DN topology and impedances. Nevertheless, when the emergency control requests reactive power injection, all DGUs in the ADN support equally. It is generally agreed that in an emergency situation being optimal is not the main goal. An emergency controller must act quickly and reliably, even at the cost of some sub-optimality. The priority is to get the necessary reactive power support (as close to the demand as possible).

Note also that there is little risk that the noise affecting voltage measurements induces misjudgment of the operating point in the $\left(V_{t}-V_{d}\right)$ space. Indeed, the relatively low speed of corrective control allows applying filters to the voltage measurements. In Refs. [12] and [13], a moving-average filter was shown to be very effective for the type of dynamics of concern in this paper.

\section{Instability detection}

In the best case, the control scheme should be able to detect the potential voltage instability without the need for an external alarm. Alternatively, the alarm could be issued by the transmission system operator, taking advantage of wide-area monitoring techniques. A survey of voltage instability detection methods was provided in [14]. The methods range from mere monitoring of low voltages to sensitivities computed from a model fitted to real-time synchrophasor measurements [13]. In this paper, two detection methods are considered. First, the Local Identification of Voltage Emergency Situations (LIVES) described in [12] is used. This method uses only $V_{d}$ and the tap changes in order to detect a developing voltage instability. The second method merely consists in comparing the measured transmission voltage $V_{t}$ with a minimum threshold.

\section{TEST SYSTEM}

The results presented in this paper are based on time-domain simulations performed on an extended version of the IEEE Nordic test system for Voltage Stability and Security Assessment, depicted in Fig. 5, detailed in [2] and implemented as reported in [3].

The extended version is a 1791-bus model and has been obtained by replacing the 11 loads of the Central area, by ADNs. A total of $144 \mathrm{ADNs}$ are connected to that region. Each ADN instance is based on the CIGRE medium-voltage network with European configuration described in [15]. DGUs and dynamic loads are considered in each DN as shown in Fig. 6. The dynamic part of the load models represents $10 \%$ of the total consumption and consists of small residential and industrial induction motors [16]. The static part is represented by an exponential model according to:

$$
\begin{aligned}
& P=P_{0}\left(\frac{V}{V_{0}}\right)^{\alpha} \\
& Q=Q_{0}\left(\frac{V}{V_{0}}\right)^{\beta}
\end{aligned}
$$

where $P_{0}, Q_{0}$ and $V_{0}$ are the initial values of the active power, reactive power and voltage, respectively. The exponents are initially set to $\alpha=1$ (constant current) and $\beta=2$ (constant impedance). By modifying these exponents, different voltage sensitivities of load can be obtained.

As for the DGUs, they supply $20 \%$ of the total load consumption in each DN. A simplified version of the WECC 


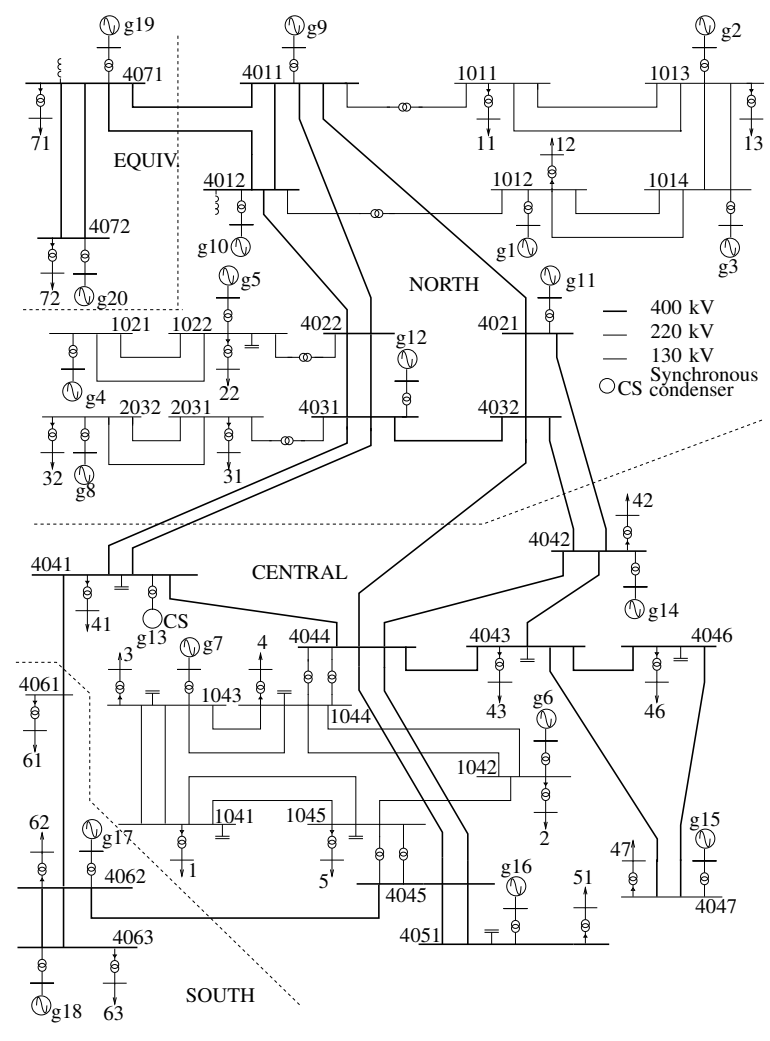

Fig. 5. Nordic test system - single line diagram.

model for inverter-based generators is used [17]; its implementation can be found in [18]. The simplification consists in omitting: 1) all dynamic components not relevant for long-term voltage stability analysis (e.g. the fast reactive current injection during system faults), and 2) all components characteristic of large plants (such as the plant-level controller). The model is set to local active and reactive power control as it is common practice for units dispersed in distribution networks. Active current is prioritized and the maximum current magnitude is set to $1.1 \mathrm{pu}$ on the DGU converter base.

The control of distribution transformer LTCs, is detailed in [2] and [3]. The time delay between consecutive tap operations has been randomized between 28 and $32 \mathrm{~s}$ for the first tap operation, and between 8 and $12 \mathrm{~s}$ for subsequent ones. This is to avoid unrealistic tap movement synchronization between the various transformers.

Each load in the central area has been replaced by a number of ADNs connected in parallel in order to match the original active power. Shunt compensation has been adjusted to match the original power factor. Therefore, the power flows in the transmission grid are left unchanged with respect to [2], [3].

Note that there is a significant voltage drop (of about $5 \%$ ) between buses 1 and 6 in the original DN [15] (see Fig. 6). In order to keep acceptable voltages at all buses, the voltage setpoint of the distribution transformer LTC has been increased to $1.05 \mathrm{pu}$. As a result, all initial voltages inside the ADNs are between 1.0 and $1.05 \mathrm{pu}$. The LTC half deadband is $0.01 \mathrm{pu}$ for all transformers.

Overall, this extension of the original IEEE Nordic test

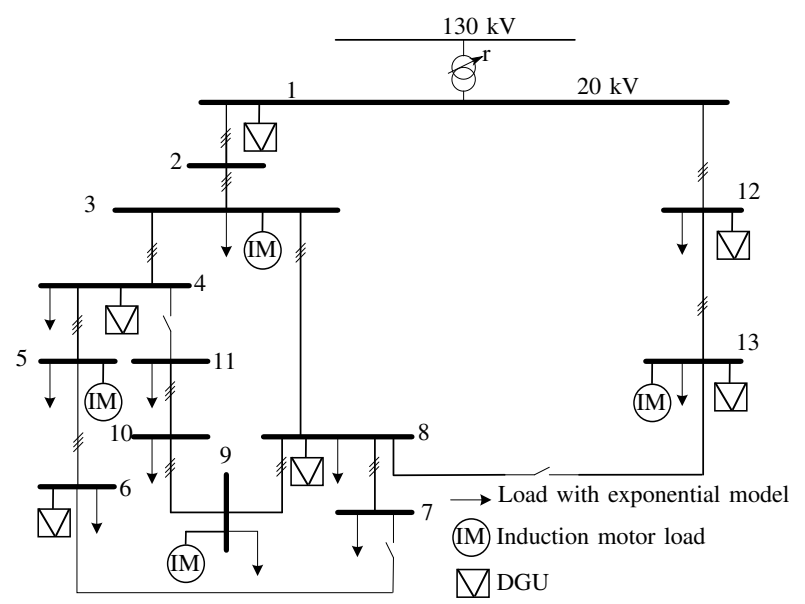

Fig. 6. Active Distribution Network - inspired of [15].

system includes 864 DGUs, 1595 loads with exponential model and 576 induction motor loads.

\section{Simulation Results}

\section{A. Emergency control settings}

The moment of alarm, at which $V_{t}^{\min }$ and $V_{d}^{\max }$ are stored, has been selected according to the two considered detection methods, i.e. if LIVES detects a developing instability or if $V_{t}$ falls below $0.95 \mathrm{pu}$. Note that such a conservative threshold would not be acceptable if the control scheme was intrusive (e.g. load shedding). As the proposed control scheme is nonintrusive, it can be used as a first line of defense.

As for the parameters $\epsilon$ and $\delta$, the horizontal deadband in Area 4 should be wide enough so $V_{d}$ does not "jump" from one side to the other after a single tap change. Therefore the value of $\epsilon$ has been set to $0.02 \mathrm{pu}$, which corresponds to the original LTC deadband. The vertical deadband can be narrower because the changes in $V_{t}$ highly depend on the more continuous DGU actions. The value of $\delta$ has been set to $0.01 \mathrm{pu}$.

Even though DGUs allow fast control actions, the DGU reactive power increase rate is intentionally limited in order to avoid abrupt changes. A rate of increase of $0.01 \mathrm{pu} / \mathrm{s}$ on the DGU MVA base has been considered.

\section{B. Categorization of transmission buses}

The proposed emergency scheme exploits the effect that a reactive power variation by the ADNs connected to a transmission bus has on its voltage. Therefore, before analyzing the results, it is of interest to characterize the transmission buses in terms of their voltage sensitivity to reactive power variations. Consider bus 1044 in Fig. 5 as an example. This bus is directly connected to the $400 \mathrm{kV}$ network through two parallel transformers. Hence, it has a relative high short circuit power compared to other buses in the Central area. As a consequence, the voltage at bus 1044 is expected to be stiff, i.e. difficult to raise by means of reactive power variations. On the other hand, buses such as 1041 (with lower short circuit power) may be more responsive to reactive power variations.

Note that emergency control actions do not take place in steady state. Dynamic components such as the OELs of 

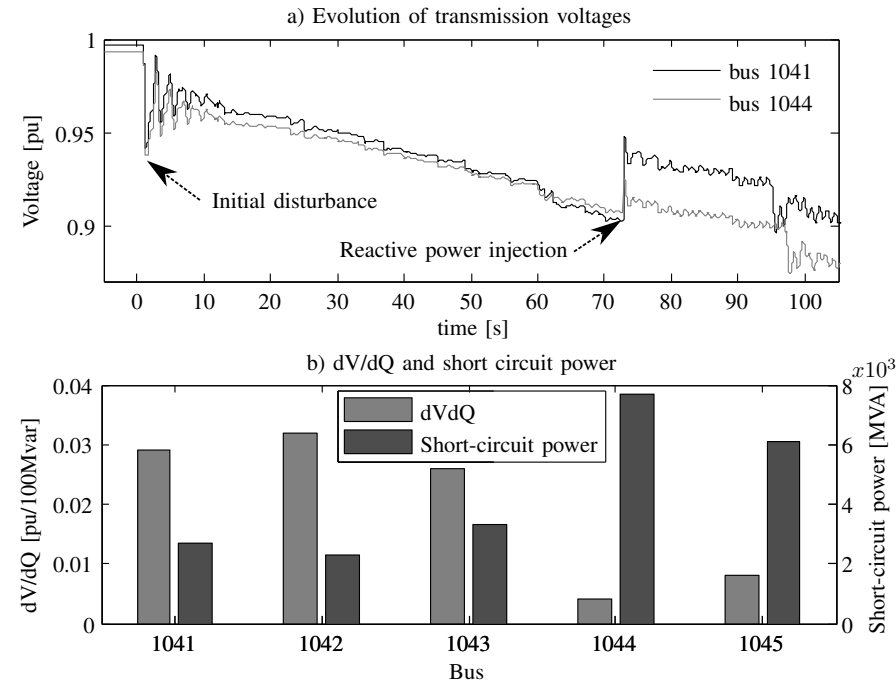

Fig. 7. Voltage sensitivities to reactive power and short circuit powers.

synchronous generators may have a non negligible impact when assessing the voltage sensitivity to reactive power. In order to take those factors into account, separate time-domain simulations have been carried out. They consist of injecting a predefined amount of reactive power (e.g. 100 Mvar) into the bus of concern and observing the resulting voltage increase. This is illustrated in Fig. 7 showing the time evolution of two representative voltages. The reactive power injection is applied when the lowest transmission voltage falls below $0.9 \mathrm{pu}$, ensuring that the effect of the relevant components will be considered. The calculated sensitivities of voltage to reactive power are shown in Fig. $7 \mathrm{~b}$ for five buses of the Central area. The corresponding short circuit powers are also shown. It can be seen that the voltages at buses 1044 and 1045 are difficult to influence with reactive power variations (low $\mathrm{dV} / \mathrm{dQ}$ and high short circuit power). Buses 1041 and 1042 show the opposite behavior. This has a significant impact on the results shown hereafter.

\section{Overview of transmission and distribution voltages}

The considered disturbance is a solid three-phase fault on line 4032-4044 (see Fig. 5), cleared in $0.1 \mathrm{~s}$ by tripping that line. This instability scenario is fully documented in [2] (see "Case A"). Five study cases are investigated as summarized in Table I. They were selected to highlight the trade-offs of the various emergency control schemes shown in Fig. 3.

Figure 8 shows the voltage evolutions at buses 1041 (high sensitivity to reactive power) and bus 1044 (low sensitivity), respectively. The horizontal dashed line shows $V_{t}^{\min }$, the transmission voltage at the moment of alarm.

Figure 9 provides examples of distribution voltage evolutions in the stabilized cases. Each subplot shows both the highest and lowest voltages inside the DN.

\section{Case 1}

In Case 1, there is no emergency control. Long-term voltage instability is driven by OELs reducing generator voltage support and LTCs trying to restore load powers [2], [11].
TABLE I

STUDY CASES

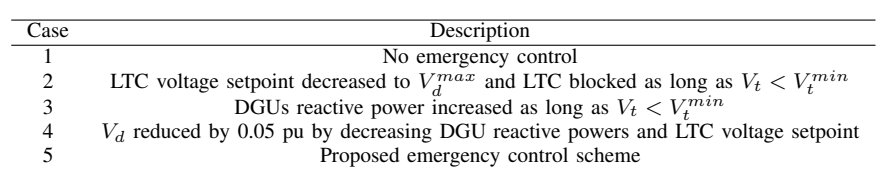

a) Voltage at bus 1041

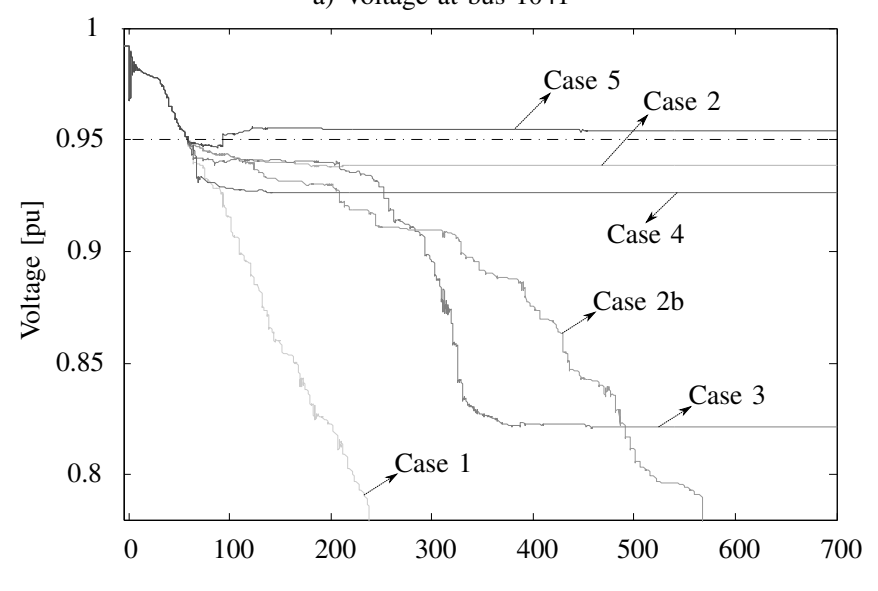

b) Voltage at bus 1044

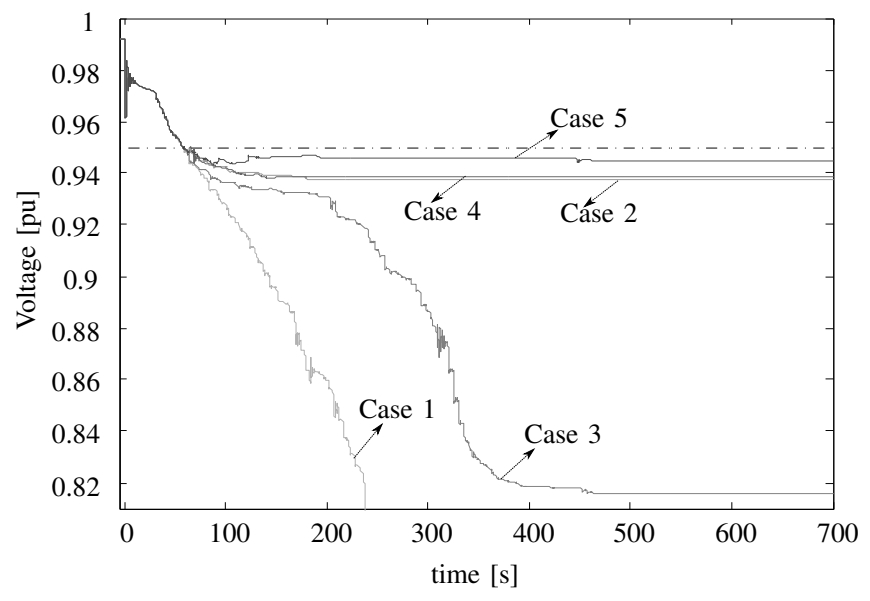

Fig. 8. Voltage evolution of buses 1041 and 1044.

\section{E. Case 2}

In Case 2, the LTC setpoints are lowered to $V_{d}^{\max }$, i.e. the value of $V_{d}$ at the moment of alarm. Furthermore, the LTCs are blocked as long as $V_{t}<V_{t}^{\min }$, where $V_{t}^{\min }$ is the value of $V_{t}$ at the moment of alarm. This somewhat combines controls 2 and 3 in Fig. 3 with the difference that $V_{d}$ is not lowered to a predefined value. Case 2 corresponds to Area 1 in Fig. 4, but without the support of DGUs.

As seen in Fig. 8, the resulting reduction of distribution voltages is not enough to keep the transmission voltages in the desired range, above $V_{t}^{\min }$. The instability mechanism is stopped. However, the reason is not the fact that the voltage setpoint was slightly lowered, but the fact that the LTC is blocked if $V_{t}<V_{t}^{\text {min }}$. The sole reduction of LTC setpoints to $V_{d}^{\max }$ is not enough to stop the instability. This can be seen in Fig. 8a as Case 2b. Thus, if the instability is counteracted by only acting on LTCs, the DN voltages are significantly 
a) Case 2

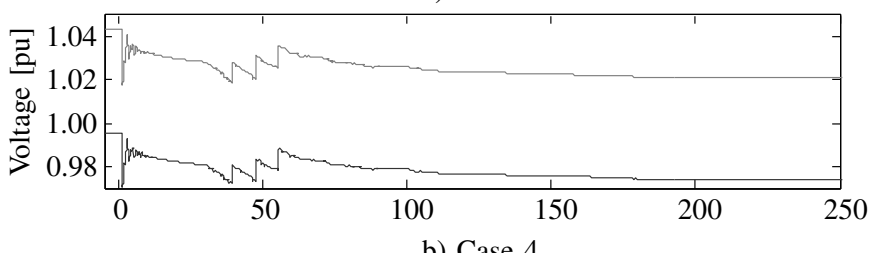

b) Case 4
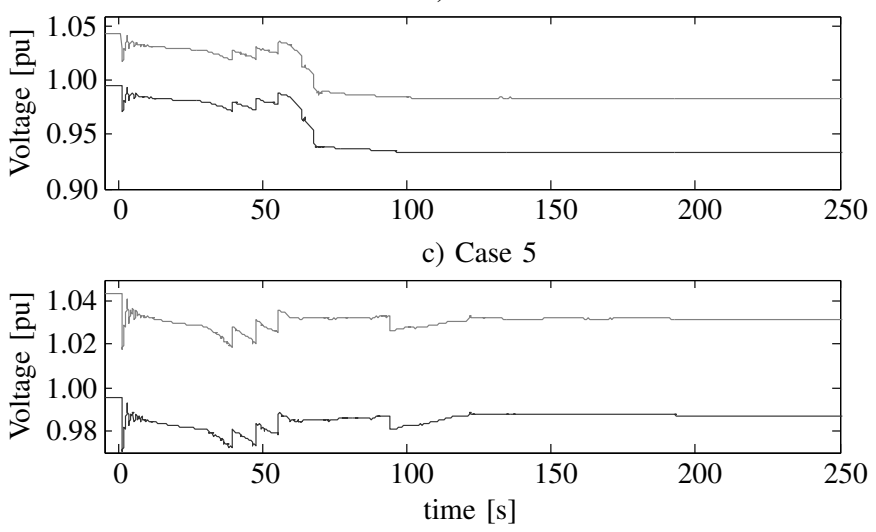

Fig. 9. Example of distribution voltage evolution in the stabilized cases.

affected by either blocking the LTC or considerably lowering its setpoint, e.g. by $5 \%$.

\section{F. Case 3}

Case 3 corresponds to control 5 in Fig. 3. More precisely, the DGUs inject reactive power as long as $V_{t}<V_{t}^{\min }$ in order to support voltage during the emergency. As seen in Fig. 8, the system is long-term unstable. Even if the system degradation does not end up in short-term instability [11] and all synchronous generators keep synchronism, transmission voltages settle to totally unacceptable values.

This result confirms that a mere reactive power injection by the DGUs may have detrimental effects on long-term voltage stability. This is confirmed by analyzing the trajectory in the power space, as shown in Fig. 10. This figure refers, as an example, to the net power of the 14 ADNs connected to bus 1041. The black dot corresponds to the pre-disturbance longterm equilibrium. The solid circle shows the post-contingency operating point before the control action and the grey dot shows the targeted equilibrium after emergency control.

Even though increasing the reactive power injection of DGUs decreases the net reactive power $Q_{t}$ seen by the transmission system, it increases the load-side voltage and, hence, the load consumption. This leads to a counterproductive increase of $P_{t}$, the net active power seen by the transmission system, and long-term voltage instability cannot be counteracted.

Note that for complex systems, such as the one used in this work and detailed in Section IV, it is impractical to calculate the actual boundary of the feasible region. However, the proposed control scheme does not require knowing said boundary. As explained in Section II, whatever the complexity of the feasible region, it still holds true that $P_{t}$ and/or $Q_{t}$ should be decreased (ideally moving downwards in the load power space) to counteract voltage instability.

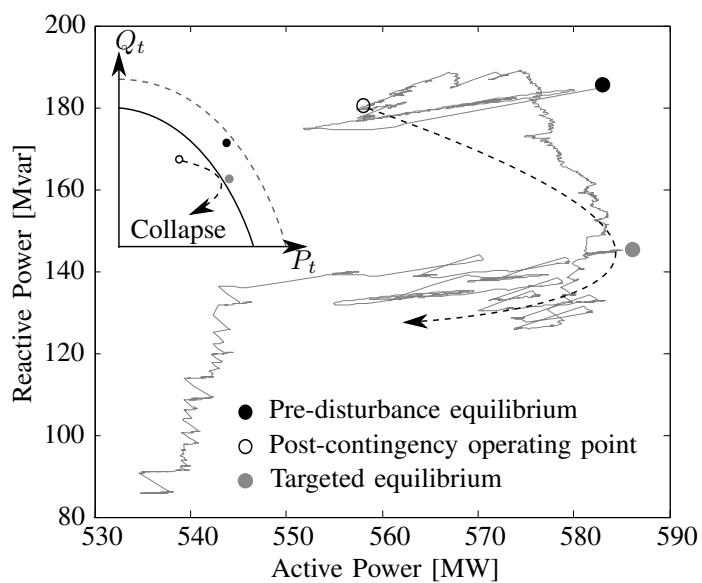

Fig. 10. Trajectory in the power space for Case 3 - Net power of the ADNs connected to bus 1041 .

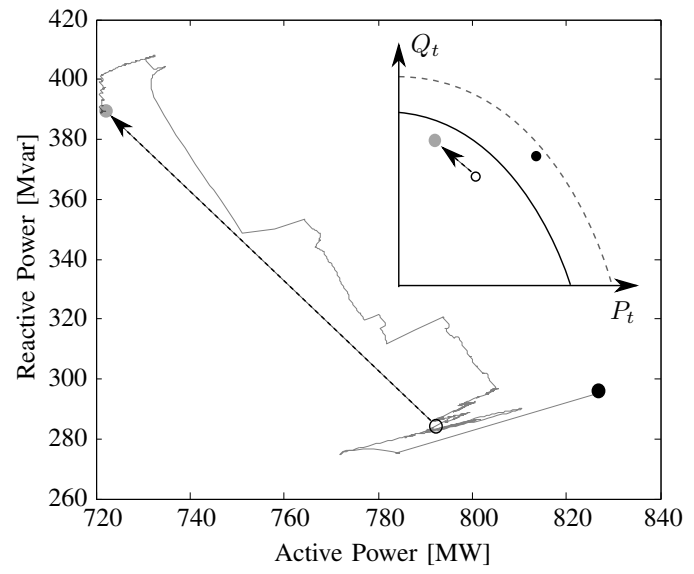

Fig. 11. Trajectory in the power space for Case 4 - Net power of the ADNs connected to bus 1044 .

\section{G. Case 4}

Case 4 is similar to the study in Refs. [6], [7]. It corresponds to control 4 in Fig. 3, in which the DGUs and the LTCs are used to reduce $V_{d}$ by a predefined value, namely $0.05 \mathrm{pu}$. In this case, the DGUs decrease or may even consume reactive power to achieve the load-side voltage reduction.

As seen in Fig. 8, this emergency action stops the instability mechanism, but the transmission voltages remain below their values at the moment of alarm. This action is successful in avoiding voltage instability. Nevertheless, there are two undesired consequences: 1) the DGUs have to reduce reactive power injection, increasing the net reactive power seen by the transmission system, and 2) the distribution voltages are significantly reduced as seen in Fig. 9b.

The trajectory in the power space is shown in Fig. 11. In this plot, the net powers of the 20 ADNs connected to bus 1044 are considered. The control action requires the DGUs to reduce their reactive power injections, which leads to a counterproductive effect of increasing the total reactive power seen by the transmission system $Q_{t}$. This suggests that in some cases this action could lead to instability if the targeted equilibrium point leaves the feasible region. 


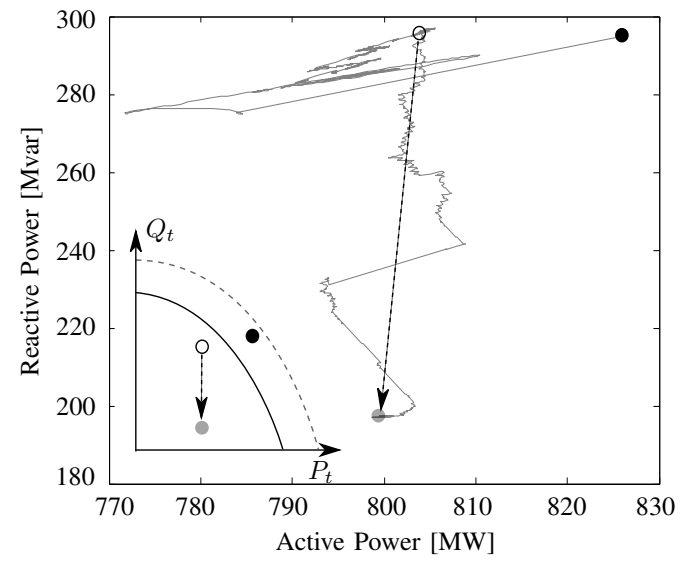

Fig. 12. Trajectory in the power space for Case 5 - Net power of the ADNs connected to bus 1044 .

\section{H. Case 5}

Case 5 illustrates the proposed emergency control scheme detailed in Section III-B. Consider first the voltage of bus 1041. As seen in Fig. 8a, the instability mechanism is stopped and the transmission voltage recovers $\left(V_{t}>V_{t}^{\text {min }}\right)$. In fact bus 1041 shows a high sensitivity to reactive power variations. Therefore, advantage is taken from the reduction of the reactive power drawn from the transmission system. This can be observed in Fig. 8a at around $t=100 \mathrm{~s}$.

Consider now the voltage of bus 1044. The trajectory in the power space is shown in Fig. 12. It shows that the reactive power support by DGUs, decreases the total reactive power $Q_{t}$ seen by the transmission system without the counterproductive effect of increasing the net active power $P_{t}$ and without affecting the distribution voltages, as seen in Fig. 9c. As a result, the operating point in the power space moves in a downwards trajectory which is definitely preferable to that shown in Fig. 11.

In this case, the control achieves one goal: the total reactive power seen by the transmission system is reduced, moving the operating point away from the border of the feasible region. Nevertheless, due to its low sensitivity to reactive power variations, the voltage at bus 1044 remains a little below the desired value, as seen in Fig. 8b. This does not satisfy the fifth design criterion in Section III-A. To better meet that criterion, the control parameters can be adjusted as follows:

- if the bus has low sensitivity to reactive power variations, decreasing $V_{d}^{\max }$ is beneficial. Priority is given to exploiting load sensitivity to voltage.

- At buses with high sensitivity to reactive power injection, increasing $V_{t}^{\text {min }}$ promotes the reactive power support by DGUs.

Figure. 13 shows that reducing $V_{d}^{\max }$ by $0.01 \mathrm{pu}$ and increasing $V_{t}^{\min }$ by 0.005 pu brings all voltages into the desired ranges.

To make the scenario more severe and validate the sixth design criterion in Section III-A, the results in Fig. 13 have been obtained assuming loads less sensitive to voltage. This is achieved by modifying the exponents $\alpha$ and $\beta$ in Eqs. 2 and 3. Namely, the exponent $\alpha$ has been reduced from 1.0 to 0.8
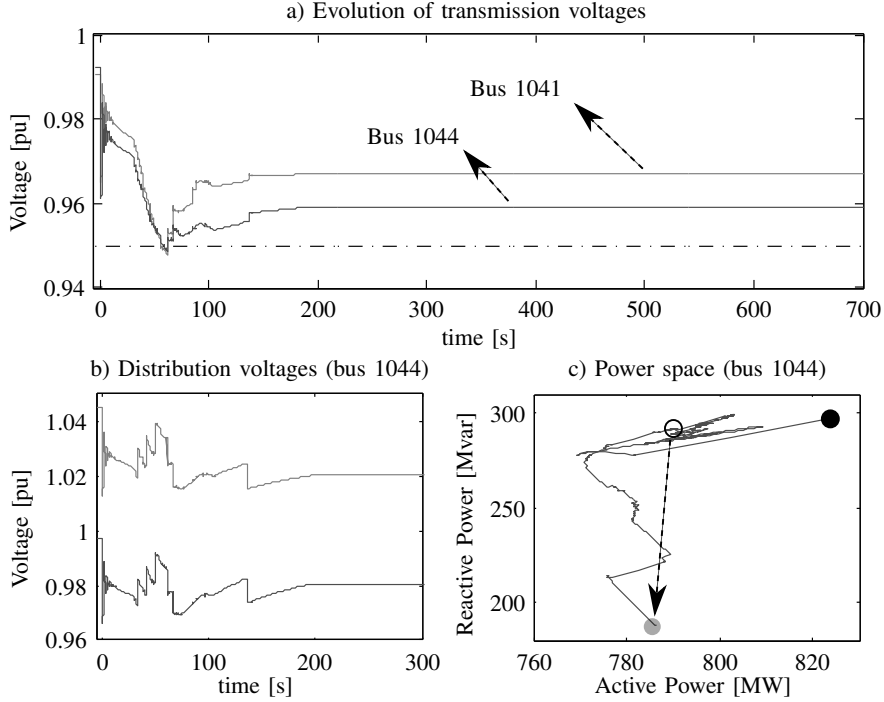

Fig. 13. Results for Case 5b - emergency control scheme accounting for the transmission voltage stiffness and the lower sensitivity of load to voltage.

and the exponent $\beta$ from 2.0 to 1.0. Note that the presence of the motor load yields an effective value of the exponent $\alpha$ lower than 0.8 for the composite load.

The control is successful regardless of the transmission voltage stiffness and the lower sensitivity of loads to voltage. Furthermore, the lowest voltage inside the ADNs is above $0.98 \mathrm{pu}$ which confirms the non-intrusive nature of the proposed control.

Note that the various ADNs are implicitly coordinated through the transmission voltage signals themselves. Indeed, when a controller acts, it contributes to increasing the voltages, which is felt by the controllers monitoring the voltages of nearby transmission buses. As a result, the latter need not act so much. Conversely, if a controller cannot contribute significantly (f.i. by lack of reactive reserves on its DGUs), it will be backed up by other controllers acting more. This coordination through voltage is made possible by the progressive nature of the controls applied and by the fact that transmission voltages react "without inertia" (unlike frequency).

\section{Limits of the controller non-intrusive nature}

In Section $\mathrm{V}-\mathrm{H}$, it was shown that the proposed control scheme can effectively support transmission voltages during emergencies even for cases of low load sensitivity to voltage. This is achieved with minimal impact on the distribution network as seen in Fig. 13.b, thus, showing the non-intrusive nature of the controller. Nevertheless, as for any scheme, there are limitations on the controller's performance that must be explored. As an example, this section studies a severe case in which the system is operating under high stress before the disturbance. This is achieved by:

- setting again the parameters of the exponential load model to $\alpha=0.8$ and $\beta=1$,

- increasing the share of induction motor loads from $10 \%$ to $35 \%$ of the total ADN power consumption, 
TABLE II

STUDY CASES FOR THE SEVERE SCENARIO

\begin{tabular}{cc}
\hline Case & Description \\
\hline C0 & No emergency control \\
C1 & Non-intrusive emergency control \\
C2 & Undervoltage load shedding \\
C3 & Non-intrusive emergency control with back-up load shedding
\end{tabular}

- correcting the power factor of induction motor loads to 0.97 instead of 1.0 .

This leads to a higher pre-disturbance reactive power demand. The synchronous generators operate at higher loading, but all below $100 \%$ of their capacity. The reactive power demand in the ADNs is higher, which can be interpreted as a lower power factor seen by the transmission system. Additionally, the load sensitivity to voltage variations is very low due to the increased share of induction motor loads and the parametrization of the exponential load model.

Four study cases are investigated as detailed in Table II. The simulation results are presented in Figs. 14 and 15.

C0: in this case, no emergency control action is applied. Long-term voltage instability is driven by OELs reducing generator voltage support and LTCs trying to restore load powers [2], [11]. The system collapses in a shorter time, namely $100 \mathrm{~s}$.

C1: In this case, the proposed control scheme is used. The controller efforts to bring $V_{t}$ above its value $V_{t}^{\min }$ at the moment of alarm are unsuccessful. At $t \cong 170 \mathrm{~s}$, the controller has been trying to restore the transmission voltages without success for two minutes and 20 seconds. At this point, several synchronous generators (g5, g6, g7, g11, g12 and g14) have lost their voltage control capability due to the action of their OELs. It is at $t=178 \mathrm{~s}$ when the system collapses with an abrupt fall of voltages. The collapse is due to the short-term dynamics becoming unstable [11] when $\mathrm{g} 8$ loses synchronism a couple of seconds after its OEL acts.

C2: In this case, the proposed emergency control is not activated. The only countermeasure is undervoltage load shedding. A simple scheme is used. When the transmission voltage falls below a threshold, e.g. $0.9 \mathrm{pu}$, the scheme starts disconnecting distribution feeders until $V_{t}$ recovers to at least $0.95 \mathrm{pu}$ (as in the previous simulations, see Fig. 8). The feeders are disconnected one by one with a pre-defined delay (typically 5 s). In this case, five feeders at bus 1044 and 4 feeders at bus 1041 are disconnected to bring $V_{t}$ to $0.95 \mathrm{pu}$. A total of $270 \mathrm{MW}$ load is disconnected from the affected area.

C3: In this case, the proposed emergency control is activated, while an undervoltage load shedding scheme is used as a backup. After one minute without success in bringing $V_{t}$ above $V_{t}^{\min }$, the load shedding scheme is activated. Two feeders at bus 1044 are disconnected for a total of $60 \mathrm{MW}$. Thus, the load shedding scheme disconnects $210 \mathrm{MW}$ less than in $\mathrm{C} 2$, which shows that the proposed controller is still less intrusive, i.e. the transmission voltages are restored to the same values with lower impact on the distribution side.

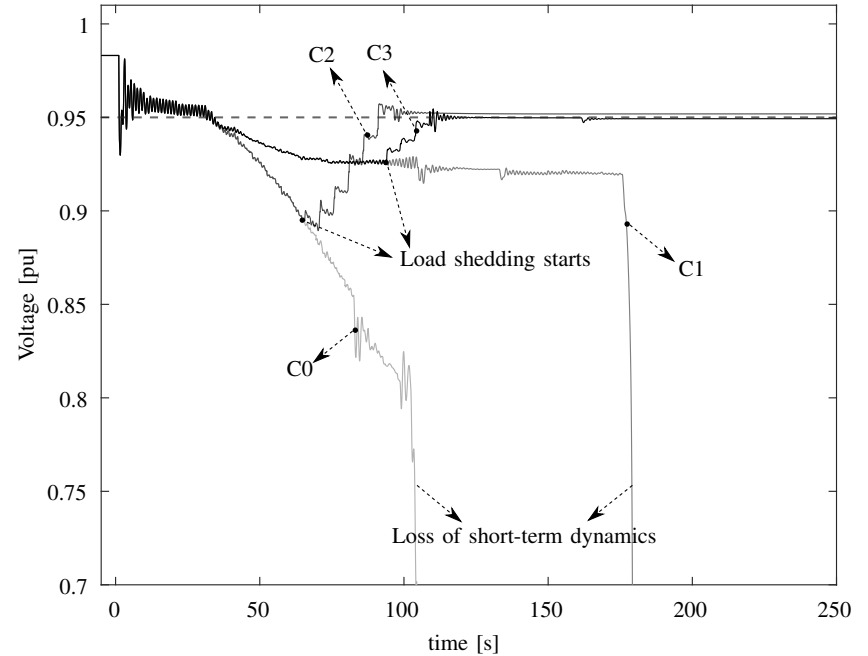

Fig. 14. Voltage evolution at bus 1044 for the severe scenario with and without load shedding scheme.

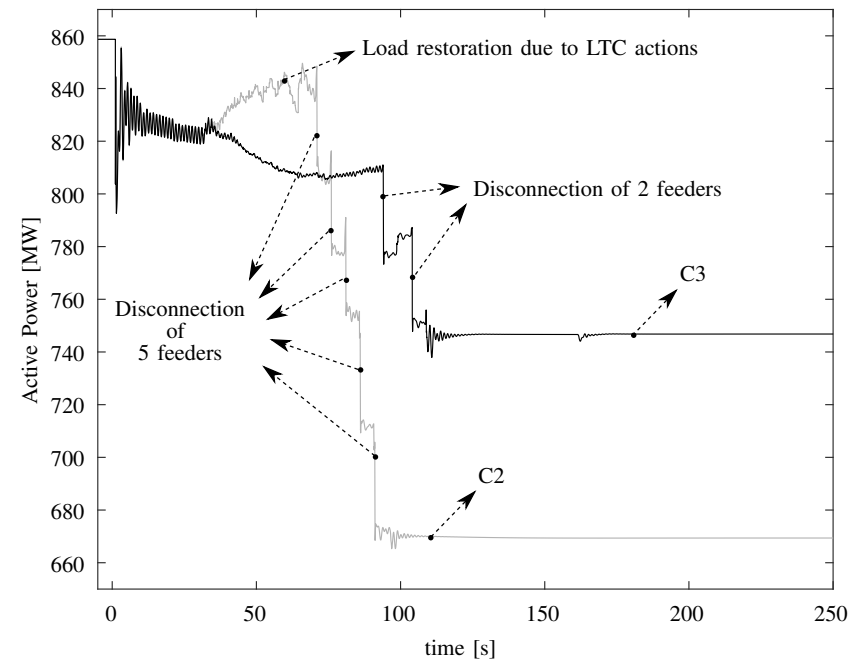

Fig. 15. Net active power of ADNs connected at bus 1044 for the cases considering load shedding.

\section{CONCLUSION}

A non-intrusive emergency voltage control involving ADNs has been proposed. The control scheme complies with the following design criteria:

1) the ADNs are minimally affected in order to support transmission voltage.

2) The control adapts to the severity of the event. Less critical situations trigger milder control actions. This avoids over-using the DSO assets.

3) The control scheme detects the emergency and acts accordingly without exchanging information outside the DN.

4) All control actions are based on two local measurements (voltage at low and high voltage side of the distribution transformer).

5) The control scheme accommodates various transmission system characteristics at the ADN point of connection in terms short circuit power or sensitivity to reactive power 
variations.

6) It also accommodates various load characteristics, i.e. type of loads and their dependency on voltage variations.

The effectiveness of the controller has been studied for different cases, including severe conditions of highly stressed operation before the disturbance. The control scheme limitations in severe situations were discussed. In such cases, it is concluded that the controller can still support transmission voltages with lower impact on the distribution network operation.

\section{ACKNOWLEDGMENT}

We acknowledge the support of our work by the German Ministry for Economic Affairs and Energy and the Projekträger Jülich within the project Netzregelung 2.0 - FKZ 0350023A. Only the authors are responsible for the content of this publication.

\section{REFERENCES}

[1] Z. Wang and J. Wang, "Review on implementation and assessment of conservation voltage reduction," IEEE Transactions on Power Systems, vol. 29, no. 3, pp. 1306-1315, May 2014.

[2] IEEE Task Force on Test Systems for Voltage Stability and Security Assessment, "Test Systems for Voltage Stability Analysis and Security Assessment," PES-TR19, Tech. Rep.,Aug. 2015.

[3] L. D. P. Ospina, A. F. Correa, and G. Lammert, "Implementation and validation of the nordic test system in digsilent powerfactory," in 2017 IEEE Manchester PowerTech, June 2017, pp. 1-6.

[4] Forum Netztechnik Netzbetrieb im VDE, "Maßnahmen zur Vermeidung spannungskritischer Netzzustände,” September 2013.

[5] H. Sun, Q. Guo, J. Qi, V. Ajjarapu, R. Bravo, J. Chow, Z. Li, R. Moghe, E. Nasr-Azadani, U. Tamrakar, G. N. Taranto, R. Tonkoski, G. Valverde, Q. Wu, and G. Yang, "Review of challenges and research opportunities for voltage control in smart grids," IEEE Transactions on Power Systems, vol. 34, no. 4, pp. 2790-2801, 2019.

[6] P. Aristidou, G. Valverde, and T. Van Cutsem, "Contribution of distribution network control to voltage stability: A case study," IEEE Transactions on Smart Grid, vol. 8, no. 1, pp. 106-116, Jan 2017.

[7] IEEE Power System Dynamic Performance Committee, "Contribution to Bulk System Control and Stability by Distributed Energy Resources connected at Distribution Network," PES-TR22, Tech. Rep.,Aug. 2017.

[8] C. Jamroen, B. Piriyanont, and S. Dechanupaprittha, "Load shedding scheme based on voltage instability index using synchrophasor data," in 2017 International Electrical Engineering Congress (iEECON), March 2017, pp. 1-4.

[9] B. Otomega, M. Glavic, and T. Van Cutsem, "Distributed undervoltage load shedding," IEEE Transactions on Power Systems, vol. 22, no. 4, pp. 2283-2284, Nov 2007.

[10] Z. Jianjun, S. Dongyu, Z. Dong, and G. Yang, "Load shedding control strategy for power system based on the system frequency and voltage stability," in Proc. 2018 China International Conference on Electricity Distribution (CICED), Sep. 2018, pp. 1352-1355.

[11] T. Van Cutsem and C. Vournas., Voltage Stability of Electric Power Systems. New York, NY, USA: Springer, 1998. ISBN 978-0-79238139-6.

[12] C. D. Vournas and T. Van Cutsem, "Local identification of voltage emergency situations," IEEE Transactions on Power Systems, vol. 23, no. 3, pp. 1239-1248, Aug 2008.

[13] M. Glavic and T. Van Cutsem, "Wide-area detection of voltage instability from synchronized phasor measurements. part ii: Simulation results," in IEEE Transactions on Power Systems, vol. 24, no. 3, 2009, pp. 14171425.

[14] - "A short survey of methods for voltage instability detection," in 2011 IEEE Power and Energy Society General Meeting, 2011, pp. 1-8.

[15] CIGRE Task Force C6.04, "Benchmark Systems for Network Integration of Renewable and Distributed Energy Resources," April 2014.

[16] IEEE Task Force on Load Representation for Dynamic Performance, "Standard load models for power flow and dynamic performance simulation," IEEE Transactions on Power Systems, vol. 10, no. 3, pp. 1302 1313, Aug 1995.

[17] Western Electricity Coordinating Council (WECC) Renewable Energy Modeling Task Force. (2014, Apr) WECC solar plant dynamic modeling guidelines. [Online]. Available: https://www.wecc.biz/Reliability/WECC Solar Plant Dynamic Modeling Guidelines.pdf

[18] G. Lammert, L. D. Pabón Ospina, P. Pourbeik, D. Fetzer, and M. Braun, "Implementation and Validation of WECC Generic Photovoltaic System Models in DIgSILENT PowerFactory," in 2016 IEEE Power \& Energy Society General Meeting, Boston, July 2016, pp. 1-5.

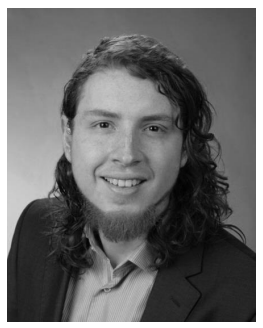

Luis David Pabón Ospina received the B.Sc. and M.Sc. degrees in electrical engineering from Universidad Pontificia Bolivariana, Medellín, Colombia, in 2011 and 2014, respectively. He received his M.Eng. degree from the University of Kempten, Kempten, Germany, in 2014. He is currently working toward the Ph.D. degree at the Dept. of Power System Control and Dynamics, Fraunhofer Institute for Energy Economics and Energy System Technology (IEE), Kassel, Germany.

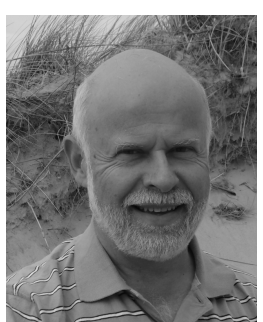

Committee
Thierry Van Cutsem (F' 05) received the M.Sc. and Ph.D. degrees from the University of Liège, Belgium, where he is currently a Research Director of the Fund for Scientific Research (FNRS) and an Adjunct Professor at the Dept. of Electrical Engineering and Computer Science. His research interests include power system dynamics, security, monitoring, control, and simulation. He has worked on voltage stability and security analysis with several TSOs in Europe and Canada. He served as Chair of the IEEE Power System Dynamic Performance 